\title{
AVALIAÇÃO DO COMPORTAMENTO DA PANI POR ESPECTROSCOPIA UV-VIS*
}

\author{
Manuela Arend Prediger ${ }^{1}$ \\ Cristiane Krause Santin ${ }^{2}$ \\ Tatiana Loiuse Avila de Campos Rocha ${ }^{3}$
}

\section{Resumo}

Este trabalho tem como objetivo investigar, através da Espectroscopia UV-Vis, os diferentes estados de oxidação da Polianilina (PAni) obtida pela síntese química, através da reação da anilina em meio ácido e persulfato de amônio como agente oxidante, sob agitação constante e baixa temperatura. A caracterização da condutividade elétrica foi determinada através da técnica de medidas de 4 pontas. Foi possível verificar a obtenção de um polímero com características condutoras, através da observação, nos Espectros UV-Vis, dos picos que caracterizam o sal esmeraldina, sendo este o estado de oxidação da PAni que apresenta propriedades condutoras. Além disso, conseguiu-se obter valores de condutividade elétrica relevantes para confirmar a obtenção da PAni condutora.

Palavras-chave: Polianilina; Espectroscopia UV-Vis; Condutividade elétrica; Síntese química.

\section{Abstract}

\section{EVALUATION OF PANI BEHAVIOR BY UV-VIS}

This study aims to investigate the different oxidation states of polyaniline (PAni) by using UV-Vis Spectroscopy. The chemical synthesis was carried out in acid medium using ammonium persulfate as oxidizing agent and the reaction was kept under constant stirring at low temperature. The electrical conductivity values were determined by using the four probe method. It was observed that a polymer with conductive characteristics was obtained. This was possible to see due to the appearance of the characteristics peaks of the emeraldine salt, in the UV-Vis spectra, which is the polyaniline oxidation state for the conductive form. Furthermore, it was verified that was possible to obtain a conductive polyaniline.

Keywords: Polyaniline; UV-Vis spectroscopy; Electrical conductivity; Chemical synthesis

1 Graduanda em Engenharia de Materiais, Bolsista de Iniciação Científica, Núcleo de Caracterização de Materiais, Universidade do Vale do Rio dos Sinos, São Leopoldo, Rio Grande do Sul, Brasil.

2 Graduada em Química, Doutora em Ciência dos Materiais, Orientadora, Núcleo de Caracterização de Materiais, Universidade do Vale do Rio dos Sinos, São Leopoldo, Rio Grande do Sul, Brasil.

3 Graduada em Química Industrial, Doutora em Ciência dos Materiais, Orientadora, Núcleo de Caracterização de Materiais, Universidade do Vale do Rio dos Sinos, São Leopoldo, Rio Grande do Sul, Brasil. 


\section{INTRODUÇÃO}

Os polímeros intrinsecamente condutores são capazes de apresentar características elétricas semelhantes aos semicondutores inorgânicos aliados à processabilidade e propriedades mecânicas de um polímero convencional. Essa interessante capacidade despertou o interesse de pesquisadores, impulsionando as aplicações desses materiais poliméricos em baterias recarregáveis, dispositivos eletrônicos, sensores químicos e térmicos, biosensores, janelas inteligentes, diodos emissores de luz, eliminação de carga estática em microeletrônica, proteção contra corrosão, recobrimento de materiais, etc. [1] Dentre os polímeros intrinsecamente condutores destaca-se a Polianilina (PAni) cujas características, como elevada estabilidade química em condições ambientais, fácil polimerização e modificação aliado ao baixo custo das matérias-primas, a tornam um polímero promissor dentro da área [1].

Os polímeros condutores apresentam cadeias com ligações duplas conjugadas, permitindo que os elétrons $\pi$ presentes $(C=C)$ sejam facilmente removidos ou adicionados a fim de formar um íon (polimérico). A transformação de um polímero com características de isolante para um semicondutor ocorre através de agentes de transferência de carga, os chamados dopantes [2].

Durante o processo de dopagem, a PAni é dopada por protonação, no qual não ocorre alteração no número de elétrons na cadeia polimérica. Essa característica particular da PAni está associada ao seu comportamento ácido-base. A PAni não dopada é classificada como base de Brönsted-Lewis e é chamada de base esmeraldina. Quando esta entra em contato com um ácido de Brönsted (doador de prótons) [3], os valores de condutividade elétrica aumentam e tornam-se superiores quando comparados com a PAni não dopada, uma vez que chegam em torno de 100 $\mathrm{S} / \mathrm{cm}[1,3]$. A dopagem química da PAni é comumente realizada em solução aquosa ácida no estado esmeraldina [1], em que se utiliza, geralmente, acido clorídrico $(\mathrm{HCl})$, por seu elevado grau de dissociação em meio aquoso e por se tratar de um ácido forte [3]. A etapa de desprotonação é realizada utilizando-se uma solução básica como o hidróxido de amônio, sendo esta uma reação reversível [4].

Fatores importantes para avaliar o grau de protonação da base são: grau de oxidação que o polímero foi sintetizado e o pH da solução dopante [1]. Os estados de oxidação da PAni apresentam valores entre 0 a 1, que é representado pela letra y. Valores de y iguais a um representam a situação em que o polímero se reduziu completamente e que contém só nitrogênios amina. Para y igual a zero, o polímero se oxidou completamente e contém só nitrogênios imina. Há, ainda, um estado intermediário, em que y é igual a 0,5 . Os três estados de oxidação diferem-se entre si pelas propriedades físicas e químicas, sendo denominados como: leucoesmeraldina $(y=1)$, esmeraldina $(y=0,5)$ e pernigranilina $(y=0)$. Dentre estes estados de oxidação, a forma esmeraldina, $50 \%$ oxidada, é a mais estável [5]. A Figura 5 apresenta a estrutura química da polianilina.

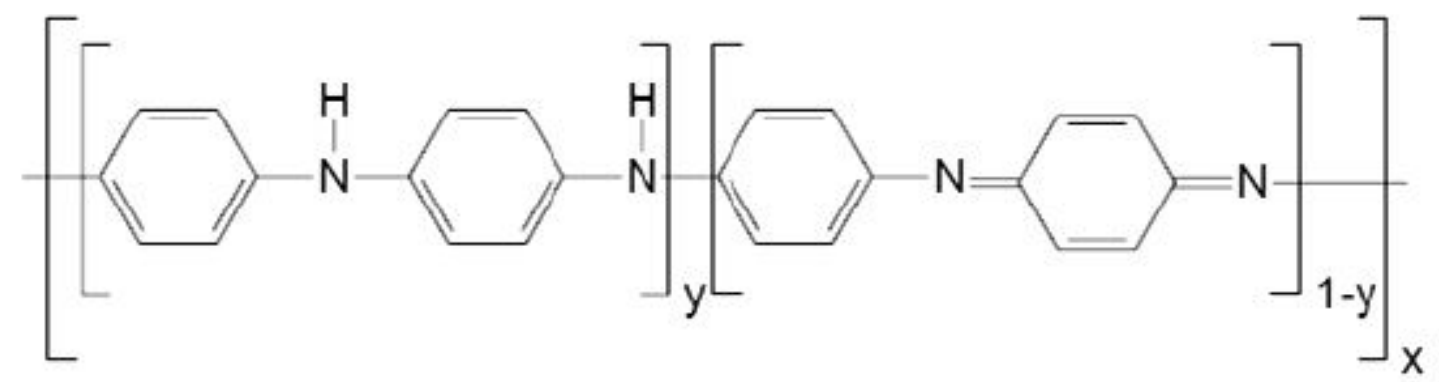

Figura 1. Estrutura Geral da Polianilina [6] 
A obtenção da PAni é realizada através de três métodos: síntese química, eletroquímica e fotoeletroquímica, sendo a síntese química a mais utilizada e vantajosa, já que possibilita a obtenção de grandes quantidades de produto final [2], além de elevada pureza [7]. O método consiste na introdução de um agente oxidante em meio acido sob agitação constante e baixas temperaturas. Diversos ácidos são utilizados para acidificar o meio, dentre esses ácidos inorgânicos cita-se $\mathrm{HCl}, \mathrm{H}_{2} \mathrm{SO}_{4}, \mathrm{HClO}_{4} \mathrm{e}$, também, ácidos orgânicos como canforsulfônico (CSA), dedocilbenzenosulfônico (DBSA) $[4,6,7]$, p-toluenosulfônico (TSA) e ácido sulfanílico (AS) [6].

Neste trabalho obteve-se a PAni a partir da síntese química e avaliou-se os parâmetros envolvidos durante o curso reacional como: temperatura, tempo reacional, tempo de adição e o método de agitação (mecânica ou magnética). $O$ produto formado foi analisado por espectroscopia UV-vis para avaliar os possíveis estados de oxidação presentes e valores de condutividade foram obtidos pela técnica de medidas de 4 pontas, com o intuito de confirmar e avaliar as análises feitas por UV-vis.

\section{MATERIAIS E MÉTODOS}

Para sintetizar a polianilina, utilizou-se anilina concentrada como monômero e ácido clorídrico $0,1 \mathrm{M}$ para acidificar o meio. Reagiu-se a anilina e o ácido clorídrico em um balão de fundo redondo, variando-se a temperatura entre $-10^{\circ} \mathrm{C}$ a $-2^{\circ} \mathrm{C}$. Dissolveu-se 0 agente oxidante (persulfato de amônio $\left(\mathrm{NH}_{4}\right)_{2} \mathrm{~S}_{2} \mathrm{O}_{8}$ ) em ácido clorídrico $0,1 \mathrm{M}$ e gotejou-se no meio reacional. A solução foi mantida sob agitação constante em agitador mecânico (aproximadamente $300 \mathrm{rpm}$ ) ou em agitador magnético por 4 horas, sendo que a cada hora coletou-se uma alíquota para análise. Posteriormente, as amostras foram filtradas e lavadas com água destilada e, secas em estufa a $50^{\circ} \mathrm{C}$. Com a síntese química concluída e a polianilina formada, mediram-se os valores de condutividade da Polianilina pelo método de quatro pontas no equipamento Cascade Microtech CS 4-64 associado a uma fonte Keithley 2400 e analisaram-se os Espectros de UV-Vis na faixa de aproximadamente 250nm a 900nm no CARY 60 com a PAni dissolvida em THF.

\section{RESULTADOS E DISCUSSÃO}

Os resultados obtidos para as amostras do Teste 5 (T5) e Teste 10 (T10) referentes a condutividade elétrica estão demostrados nas Figuras 2 e 3 . Na Figura 2, apresenta-se as condutividades das amostras coletadas no Teste 5 , em que se utilizou agitador magnético para manter a agitação constante. Observa-se uma grande variação na condutividade que, na medida em que o tempo aumentou, a condutividade elétrica diminuiu. Nos espectros UV-Vis dessa amostra percebeu-se picos menos intensos em aproximadamente 400nm e $860 \mathrm{~nm}$ (bandas características da PAni condutora) e esses picos também diminuíram com o aumento do tempo. Já no Teste 10, Figura 3, no qual se usou agitação mecânica, é possível observar que em 2 horas obtém-se um valor máximo de condutividade elétrica de $1,23.10^{-3} \mathrm{~S} / \mathrm{cm}$, o qual permanece constante e, portanto, não é influenciado pelo tempo reacional. 


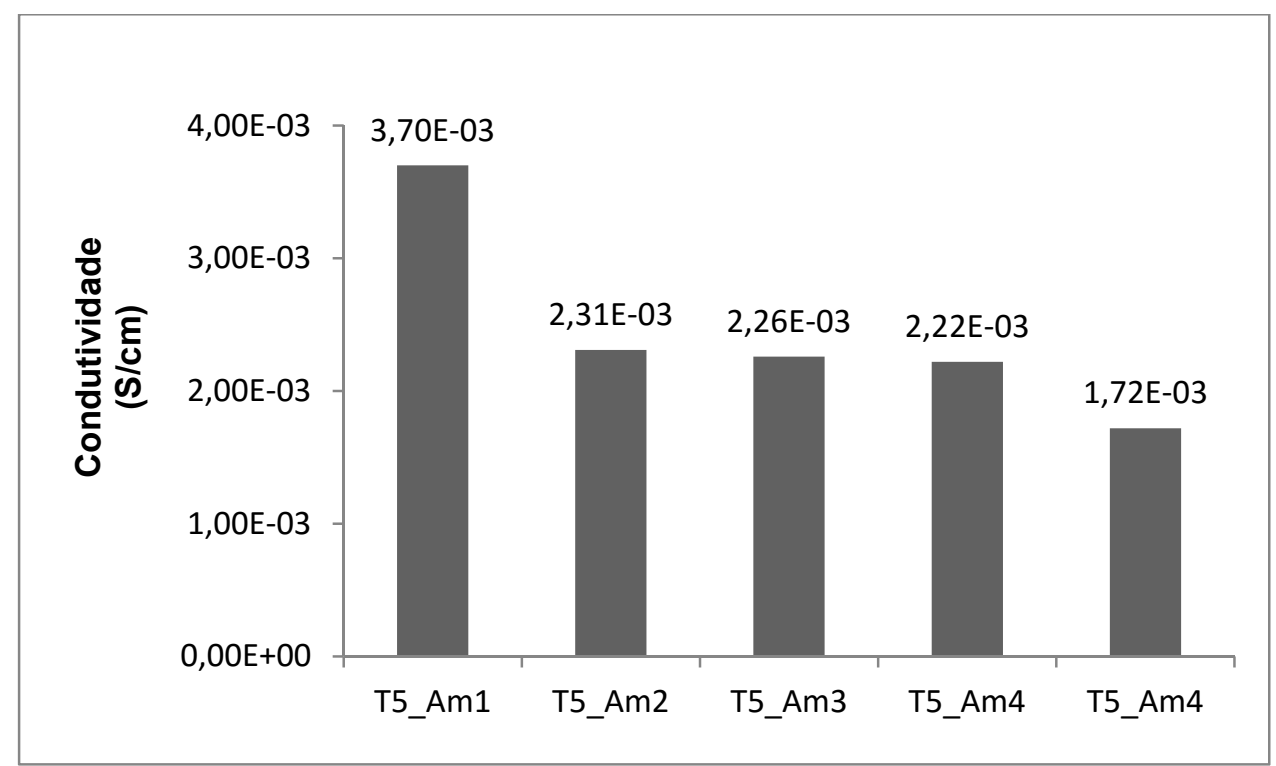

Figura 2. Condutividades do Teste 5

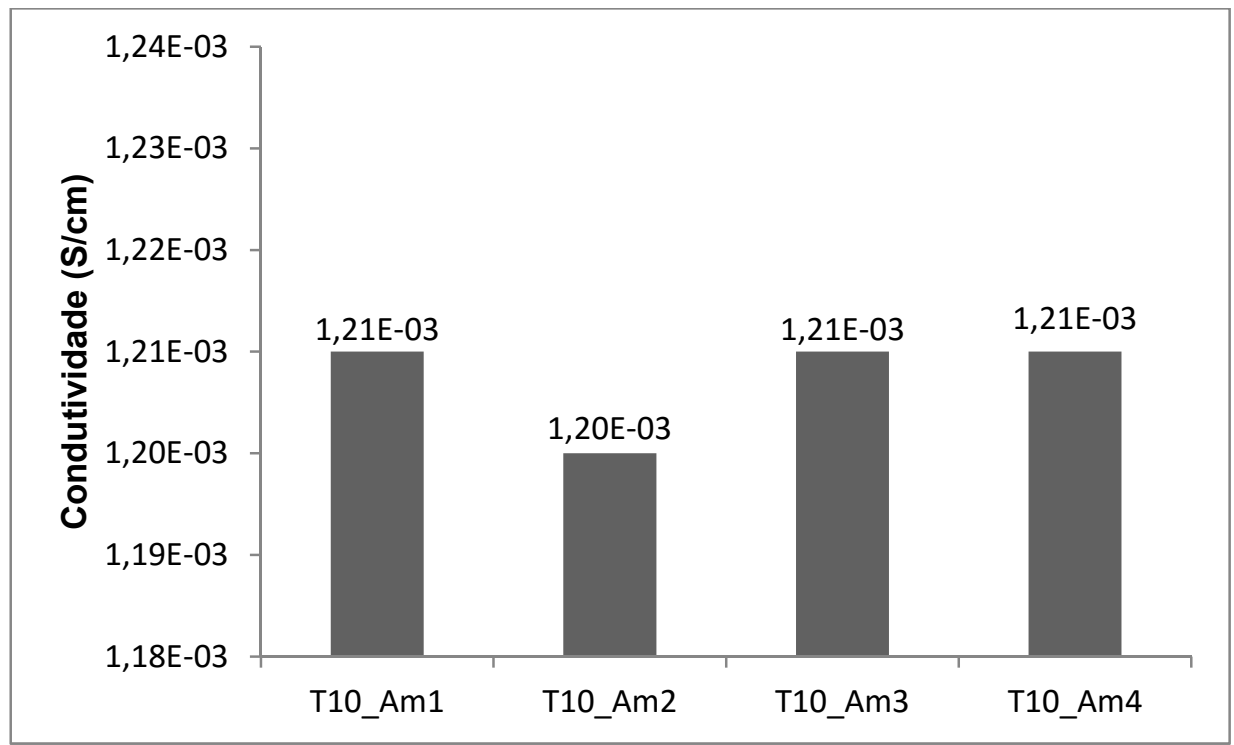

Figura 3. Condutividades do Teste 10

Estudos relatam que a Pani base esmeraldina (isolante) apresenta duas bandas: uma a aproximadamente $328 \mathrm{~nm}$, que indica a transição $\pi-\pi$ e outra a $640 \mathrm{~nm}$ que indica transições $n-\pi$. Porém, quando a base esmeraldina é dopada, observa-se que a banda localizada em $640 \mathrm{~nm}$ não aparece mais, já que essa banda está associada com as transições nos anéis quinoides que não estão presentes no sal esmeraldina (PAni condutora). Ainda, a literatura descreve a presença de duas bandas para a Polianilina dopada (aproximadamente $420 \mathrm{~nm}$ e $860 \mathrm{~nm}$ ), que correspondem às bandas polarônicas [8,9]. Em nossos estudos, foi possível observar que o Teste 10, Figura 4, apresentou picos característicos do sal esmeraldina na banda $860 \mathrm{~nm}$ e $328 \mathrm{~nm}$, porém em $420 \mathrm{~nm}$ não foi possível visualizar a formação de banda. Segundo E. S. Medeiros et al [3], quando se realiza a dopagem da PAni e o grau de dopagem supera $50 \%$, certos sítios amina atingem a protonação e certos sítios iminas continuam desprotonados por causa do nível de dopagem ser reduzido. Isso quer dizer que a dopagem da PAni não é considerada homogênea.

Já na Figura 5, observa-se a presença de uma banda de absorção na região a 640 $\mathrm{nm}$, indicando que a polianilina possui característica isolante, chamada de base 
esmeraldina. Além disso, seu pH esteve entre 8 e 9 e sua cor alterou para azul, o que confirma o processo de desdopagem realizado.

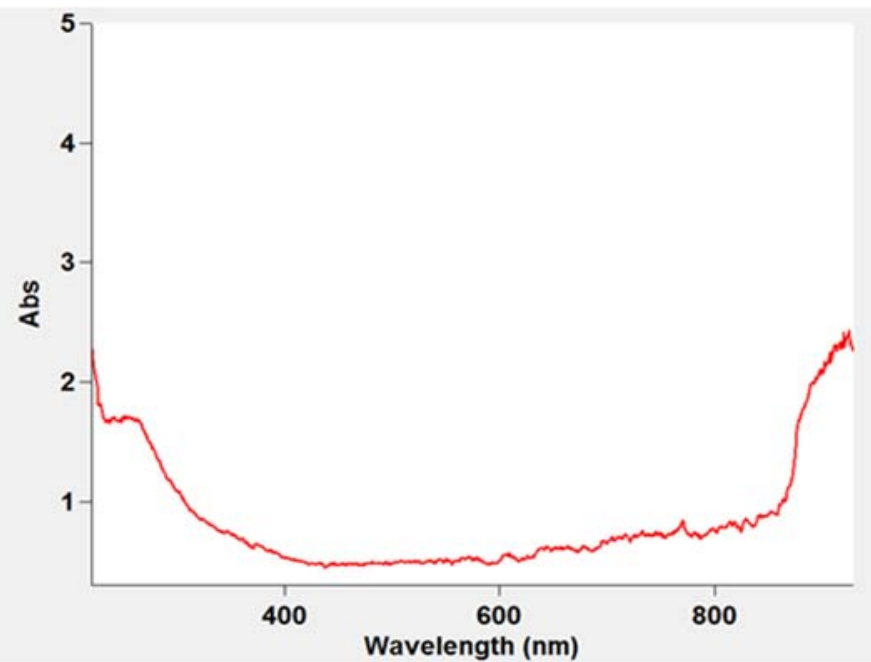

Figura 4. Espectroscopia UV-vis da Polianilina desdopada

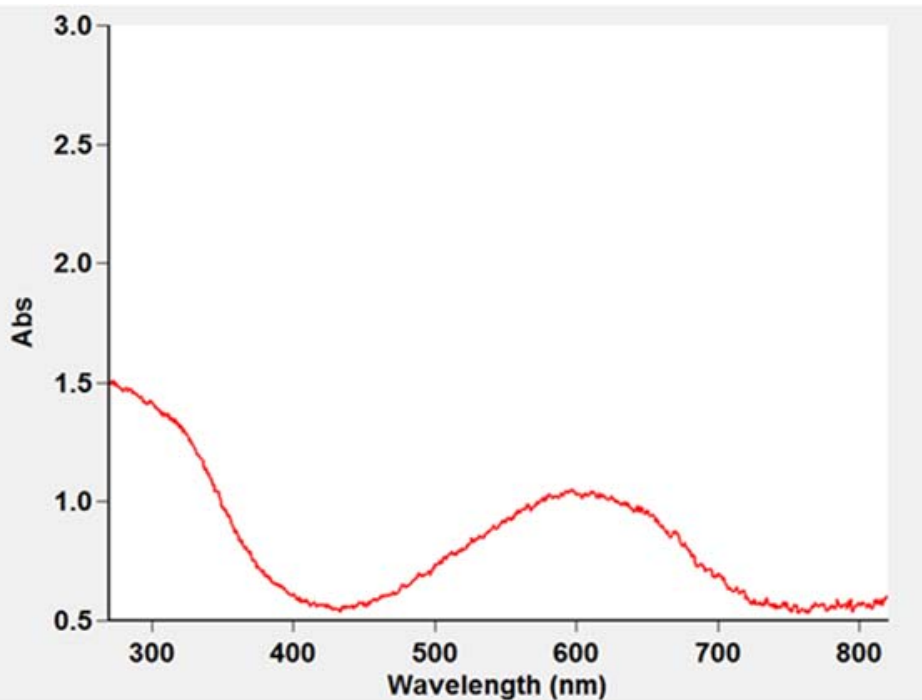

Figura 5. Espectroscopia UV-vis da Polianilina

\section{CONCLUSÃO}

A partir dos estudos feitos, conclui-se que o tempo de adição bem como o tempo reacional não possui relevância nos valores de condutividade elétrica da polianilina. Já o tipo de mecanismo utilizado para manter a agitação constante fez com que os valores de condutividade elétrica variassem. A agitação mecânica permitiu um maior controle dos valores de condutividade, uma vez que estes permaneceram constantes nas amostras coletadas. Em contrapartida, com a agitação magnética, houve considerável variação entre as amostras de um mesmo teste, o que gera incerteza nos valores na medida em que o tempo aumenta.

Portanto, o polímero condutor polianilina foi obtido com êxito, uma vez que se alcançou valores de condutividade elétrica satisfatórios e conseguiu-se analisar os parâmetros envolvidos na síntese química da polianilina. Além disso, a avaliação feita pela Espectroscopia UV-vis reforçou que a polianilina obtida é a condutora, já que foi possível observar duas bandas de absorção características do sal 
esmeraldina. E ainda foi possível, a partir da Espectroscopia de UV-Visível, monitorar a formação dos diferentes estados de oxidação da PAni.

\section{Agradecimentos}

Os autores agradecem ao Conselho Nacional de Pesquisa (CNPq), Fundação de Amparo à Pesquisa do Rio Grande do Sul (FAPERGS) e Conselho Pesquisa Superior (CAPES).

\section{REFERÊNCIAS}

1 Mattoso, L.H. “Polianilinas: Síntese, estrutura e Propriedades” Quimica Nova, 1996, 19 (4), 388-399.

2 FAEZ, Roselena; REIS, Cristiane; DE FREITAS, Patrícia Scandiucci; KOSIMA, Oscar K.; RUGGERI,Giacomo; DE PAOLI, M.-A. Polímeros Condutores, Química Nova na Escola, 2000, N. 11.

3 MEDEIROS, E. S.; OLIVEIRA, J. E. ; CONSOLIN-FILHO, N.; PATERNO, L. G.; MATTOSO, L. H. C. Uso de Polímeros Condutores em Sensores. Parte 1: Introdução aos Polímeros Condutores, Revista Eletrônica de Materiais e Processos, 2012, v.7, n.2, p. $62-77$

$4 \quad$ NEVES, Silmara das. Propriedades Fotoeletroquímicas da Polianilina. Brasil, 1997. Tese (Doutorado) - Universidade Estadual de Campinas, Campinas, 1997.

5 HANSEN, Betina. Metodologia para Produção de Biossensores Amperométricos Enzimáticos Utilizando Polímeros Condutores: Caso Polianilina. Brasil, 2011. Dissertação (Mestrado em Engenharia) - Programa Pós-graduação em Engenharia de Minas, Metalúrgica e Materiais, Universidade Federal do Rio Grande do Sul, Porto Alegre, 2011

6 MÜLLER, Franciélli. Utilização de polianilina em matriz de polímero convencional para produção de membranas catiônicas: síntese, caracterização e aplicação. Brasil, 2009. Dissertação (Mestrado em Engenharia) - Programa Pós-graduação em Engenharia de Minas, Metalúrgica e Materiais, Universidade Federal do Rio Grande do Sul, Porto Alegre, 2009.

7 SANTOS, Herisson Ferreira dos. Estudo das interações entre polianilina e semicondutores nanocristalinos coloidais de CdTe e CdSe. Brasil, 2013. Dissertação (Mestrado em Engenharia Mecânica) - Universidade Federal de São João del-Rei, São João del-Rei, 2013.

8 MARTIN, Eliza Sbrogio. Síntese e Caracterização de Blendas de Polianilina com Látex de Borracha Natural. Brasil, 2008. Dissertação (Mestrado em Ciência dos Materiais) Universidade Estadual Paulista, São Paulo, 200.

9 RODRIGUES, Paula Cristina. Síntese, caracterização e correlações estrutura/propriedades de redes mistas de polianilina/poliuretano com arquitetura molecular pré-desenhada. Brasil, 2004. Tese (Doutorado em Ciências) - Programa de Pós-Graduação em Química, Universidade Federal do Paraná, Curitiba, 2004. 